\title{
Silicon Nanowire Based MOSFET Parameter Analysis
}

\author{
${ }^{1}$ P. S. Raja, ${ }^{2}$ R. Joseph Daniel, ${ }^{3}$ Bino. N \\ ${ }^{1,2,3}$ Dept. of E \& I Engineering, Annamalai University, Annamalainagar, 608002, Tamilnadu, India.
}

\begin{abstract}
As the conventional silicon metal-oxide-semiconductor field-effect transistor (MOSFET) approaches its scaling limits many novel device structures are being extensively explored. Among them, the silicon nanowire transistor (SNWT) has attracted broad attention from both the semiconductor industry and academia. To understand device physics in depth and to assess the performance limits of SNWTs, simulation is becoming increasingly important. The objectives of this paper are to assess the performance of SNWTs. A nano device simulator called fettoy was utilized for this purpose. The various output parameters analyzed were Quantum capacitance vs. gate voltage, Drain current vs. gate voltage, drain current vs. drain voltage, Drain induced barrier lowering, Threshold swing, Carrier injection velocity, on current, off current, Output conductance, Transconductance and Voltage gain for variation in input parameters like Dielectric materials, Thickness of dielectric material, Diameter of silicon nanowire and Gate Control Parameter. Also the effect of temperature was also studied.
\end{abstract}

\section{Introduction}

As the MOSFET gate length enters the nanometer regime, short channel effects (SCEs), such as threshold voltage $\left(V_{T}\right)$ rolloff and Drain-Induced-Barrier-Lowering (DIBL), become increasingly significant, which limits the scaling capability of planar bulk or Silicon-On-Insulator (SOI) MOSFETs [1]. At the same time, the relatively low carrier mobility in silicon (compared with other semiconductors) may also degrade the MOSFET device performance (e.g., ON-current and intrinsic device delay). For these reasons, various novel device structures and materials - silicon nanowire transistors, carbon nanotube FETs, new channel materials (e.g., strained silicon, pure germanium), molecular transistors, etc all are being extensively explored. Among all these promising post-CMOS structures, the silicon nanowire transistor (SNWT) has its unique advantage - the SNWT is based on silicon, a material that the semiconductor industry has been working on for over thirty years; it would be really attractive to stay on silicon and also achieve good device metrics that nanoelectronics provides. As a result, the silicon nanowire transistor has obtained broad attention from both the semiconductor industry and academia.

Semiconductor Nano Wires (NWs) have attracted significant interest because of their potential for a variety of different applications, including logic and memory circuitry, photonics devices, and chemical and biomolecular sensors. Although many different types of semiconductor NW have been investigated, silicon NWs have become prototypical nanowire because they can be readily prepared, the $\mathrm{Si} / \mathrm{SiO} 2$ interface is chemically stable, and Si NWs are utilized in a number of device demonstrations that have well-known silicontechnology-based counterparts. Various techniques have been developed to synthesize semiconductor NWs, including the materials method known as Vapor-Liquid-Solid (VLS) growth and the templating method known as super lattice nanowire pattern transfer (SNAP).

\section{Silicon Nanowire MOSFET}

A nanowire is a wire having its diameter of the order of a nanometer (10-9 meter). At these scales, quantum mechanical effects are important hence such wires are also known as 'quantum wires'[2]. The nanowires could be used, in the near future, to link tiny components into extremely small circuits. Typical nanowires exhibit aspect ratios (length-to-width ratio) of 1000 or more. Silicon Nanowires (SiNWs) are currently attracting much attention as promising components for future nano-electronic devices such as nanowire based field effect transistors, photonic and optoelectronic devices, nanowire based solar cells and NW based chemical or biological sensors [3]. This is due to the fact that they show electronic, optical, chemical, mechanical, and thermal properties that differ from their bulk counterparts. Bulk Si is an indirect gap material, the SiNWs are useful one-dimensional nanostructure for tailoring its physical properties from indirect to direct band gap. 


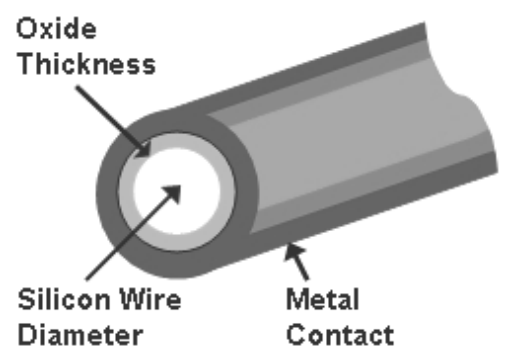

Fig.1. Structure of Silicon Nanowire transistor

Silicon nanowire transistors with various types of cross-sections are being extensively explored by a number of experimental groups. The various cross sections are the cylindrical wire (CW), triangular wire (TW) and rectangular wire $(\mathrm{RW})$ nanowire FET as shown in fig 2 , where $T_{S i}$ is the silicon body thickness, $W_{S i}$ is the silicon body width and $W_{\text {Wire }}$ is the wire width. For the CW FET, $T_{S i}=W_{S i}$ is equal to the diameter of the circular $\mathrm{Si}$ body. Triangular wire nanowire FET device was built on a Si (001) wafer and the Si body layer was etched along the (111) surfaces, so the cross-section of the Si body becomes an isosceles triangle and the channel of the device is $\langle 110\rangle$ oriented. At the same time, different types of tri-gate/gate-all-around FETs were fabricated by using wires with rectangular cross-sections. The diameters of these wire transistors are around 30-100nm and the gate lengths are $>50 \mathrm{~nm}$. In addition, we also have the cylindrical SNWT, a gate-all around structure, which offers the optimum gate control and scaling potential among all kinds of SNWTs.

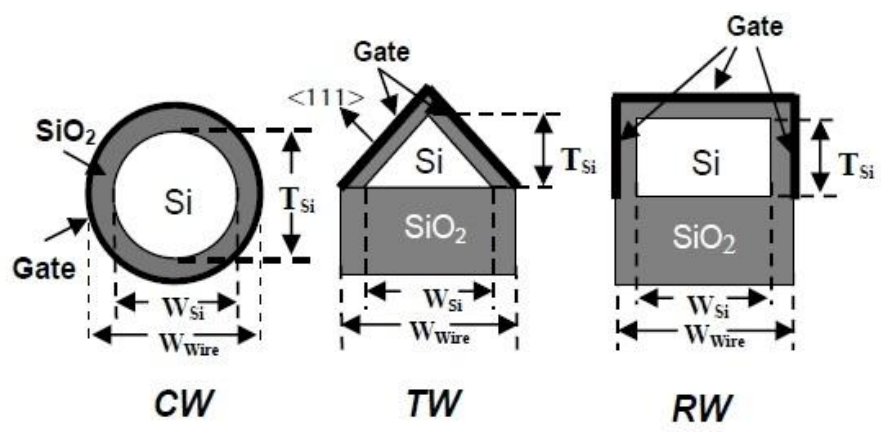

Fig. 2 The cross-sections of the various nanowire FET structures - the cylindrical wire (CW), triangular wire (TW) and rectangular wire (RW) FETs.

\section{Simulation Results and Discussion}

The various output parameters were studied by varying the input parameters as shown in the Table.1

Table .1 Various input parameters and their range of variations.

\begin{tabular}{|l|l|l|l|l|l|l|}
\hline Input parameters & \multicolumn{7}{|c|}{ Variation in parameters } \\
\hline $\begin{array}{l}\text { Dielectric material } \\
\text { (dielectric constant) }\end{array}$ & $\begin{array}{l}\text { Teflon } \\
(2.1)\end{array}$ & $\begin{array}{l}\text { Polyethylene } \\
(2.25)\end{array}$ & SiO2 (3.9) & ZrO2 (15) & $\begin{array}{l}\mathrm{HfO} 2 \\
(25)\end{array}$ & $\begin{array}{l}\text { TiO2 } \\
(40)\end{array}$ \\
\hline Dielectric thickness & $1.5 \mathrm{~nm}$ & $3 \mathrm{~nm}$ & $6 \mathrm{~nm}$ & $9 \mathrm{~nm}$ & & \\
\hline Diameter of SNW & $1 \mathrm{~nm}$ & $5 \mathrm{~nm}$ & $10 \mathrm{~nm}$ & $15 \mathrm{~nm}$ & & \\
\hline $\begin{array}{l}\text { Gate control } \\
\text { parameter (GCP) }\end{array}$ & 0.1 & 0.3 & 0.5 & 0.8 & 0.88 & 0.92 \\
\hline Temperature & $273 \mathrm{~K}$ & $300 \mathrm{~K}$ & $325 \mathrm{~K}$ & $350 \mathrm{~K}$ & $375 \mathrm{~K}$ & $400 \mathrm{~K}$ \\
\hline
\end{tabular}

\subsection{Quantum capacitance Vs Gate voltage}

The Simulations were carried out to obtain the quantum capacitance for a sweep in gate voltage ( 0 to $1 \mathrm{~V})$ in a silicon nanowire MOSFET with Drain voltage as $1 \mathrm{~V}$ and variations in input parameters. 


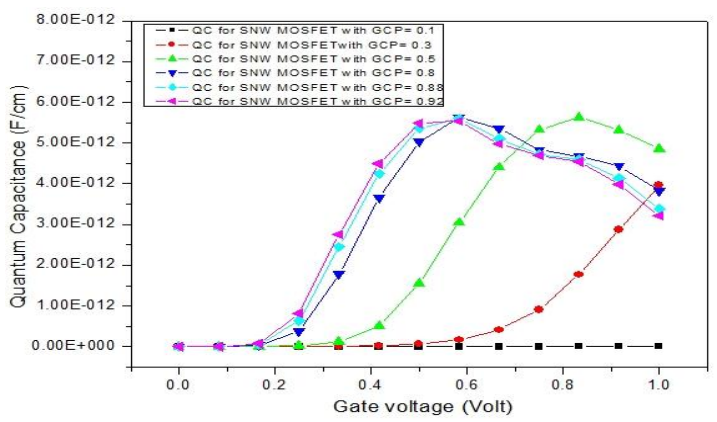

Fig.3. (a) Variation in Gate control parameter

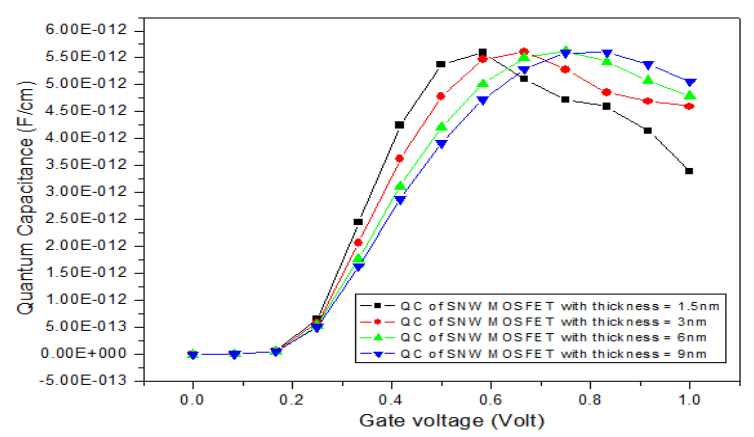

Fig.3. (c) Variation in Dielectric thickness

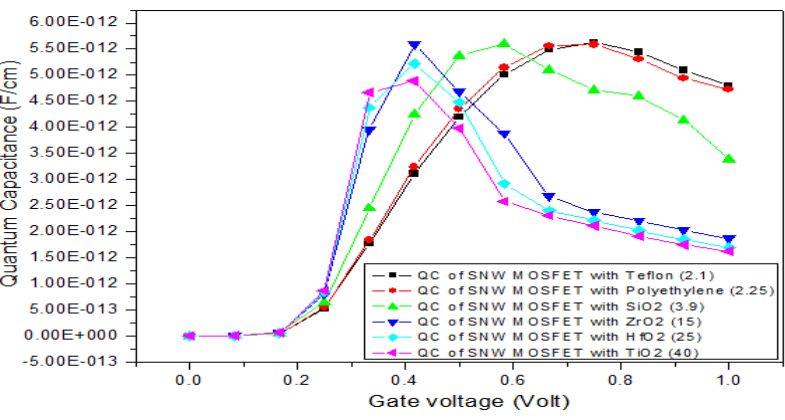

Fig.3. (b) Variation in Dielectric material

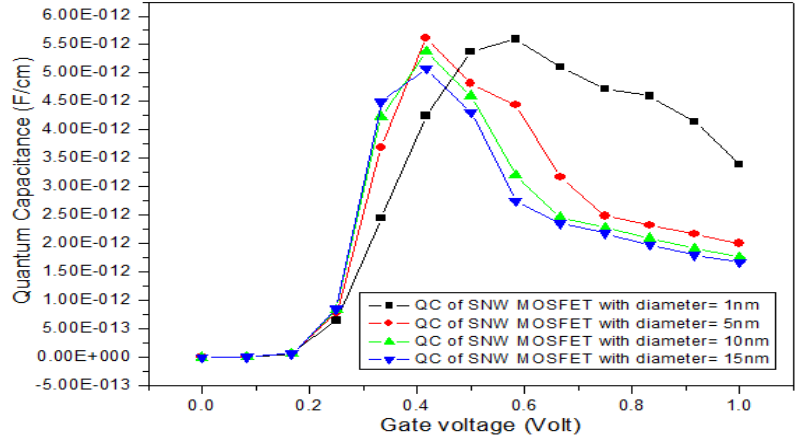

Fig.3. (d) Variation in SNW diameter

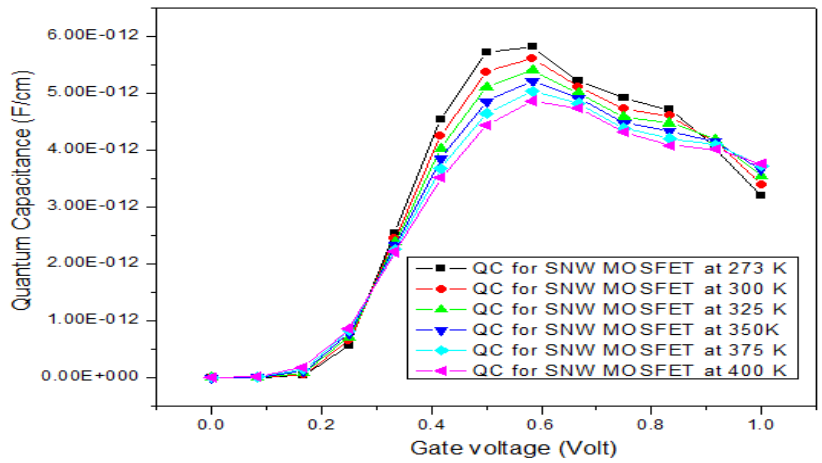

Fig.3. (e) Variation in temperature

The simulation results shows that Quantum Capacitance decreases with decrease in GCP, high-k dielectric materials, decrease in dielectric thickness, with increase in SNW diameter and with increase in temperature.

\subsection{Drain current Vs Gate voltage}

Simulations were carried out to obtain Drain current for a sweep in gate voltage ( 0 to $1 \mathrm{~V}$ ) in a silicon nano wire MOSFET with Drain voltage as $1 \mathrm{~V}$ and variations in input parameters.

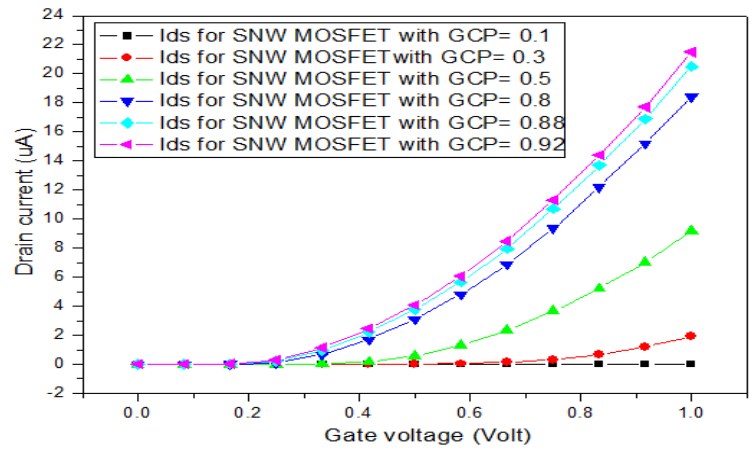

Fig.4. (a) Variation in Gate control parameter

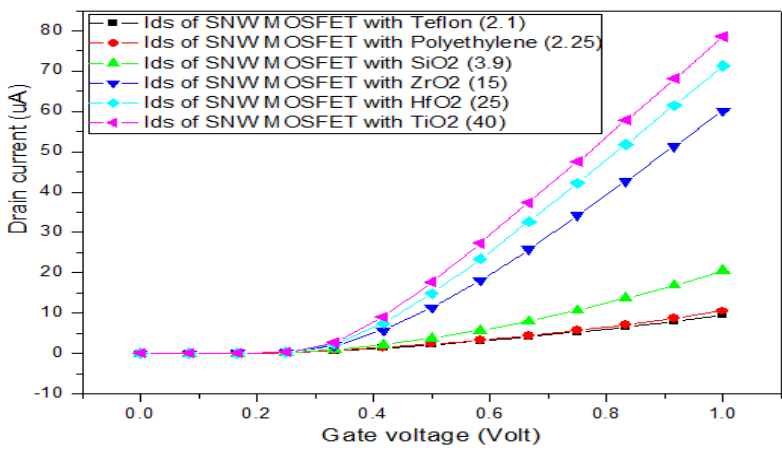

Fig.4. (b) Variation in Dielectric material 


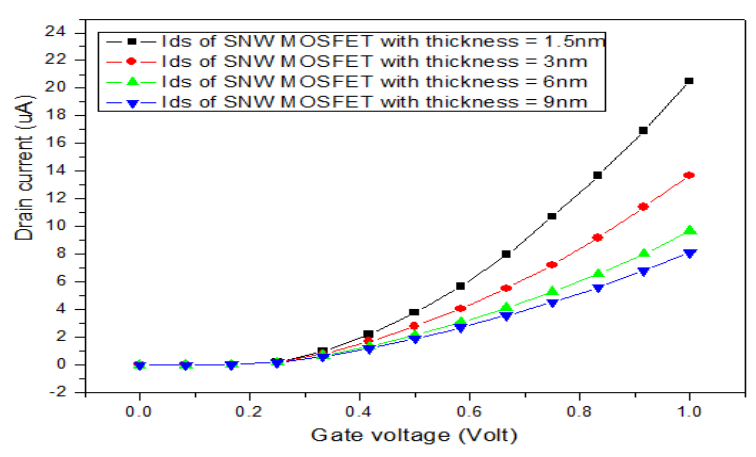

Fig.4. (c) Variation in Dielectric thickness

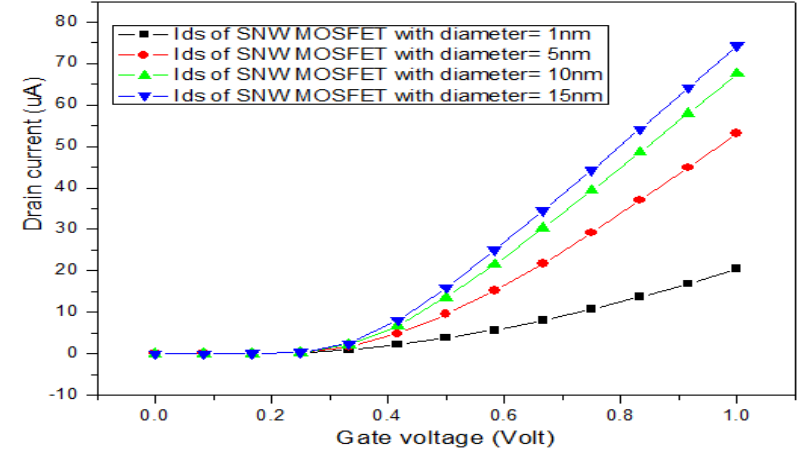

Fig.4. (d) Variation in SNW diameter

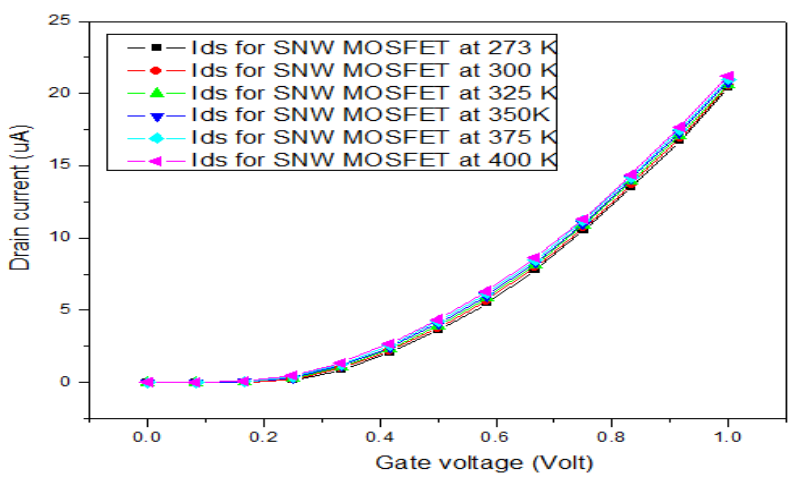

Fig.4. (e) Variation in temperature

The simulation results shows that the drain current increases with increase in GCP, high-k dielectric materials, with decrease in dielectric thickness, with increase in SNW diameter and there is no appreciable variation for change in temperature.

\subsection{Drain current Vs drain voltage}

Simulations were carried out to obtain Drain current for a sweep in drain voltage ( 0 to $1 \mathrm{~V})$ in a silicon nano wire MOSFET with Gate voltage as $1 \mathrm{~V}$ and variations in input parameters as follows

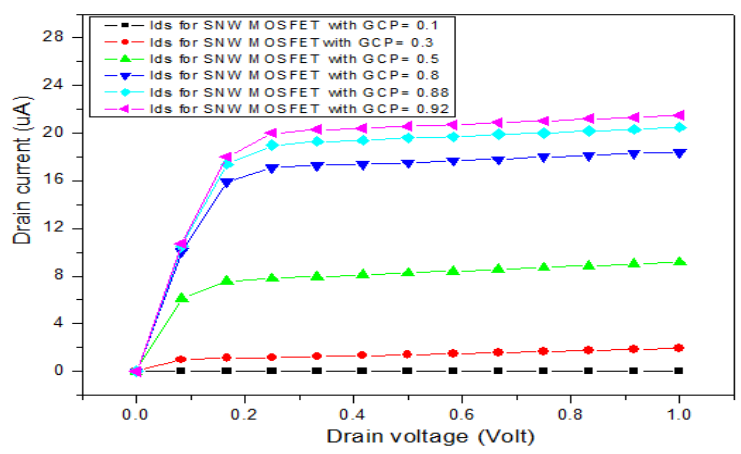

Fig.5. (a) Variation in Gate control parameter

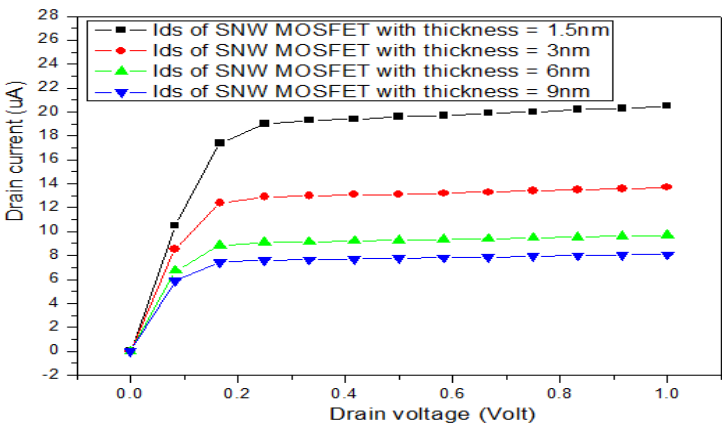

Fig.5. (c) Variation in Dielectric thickness

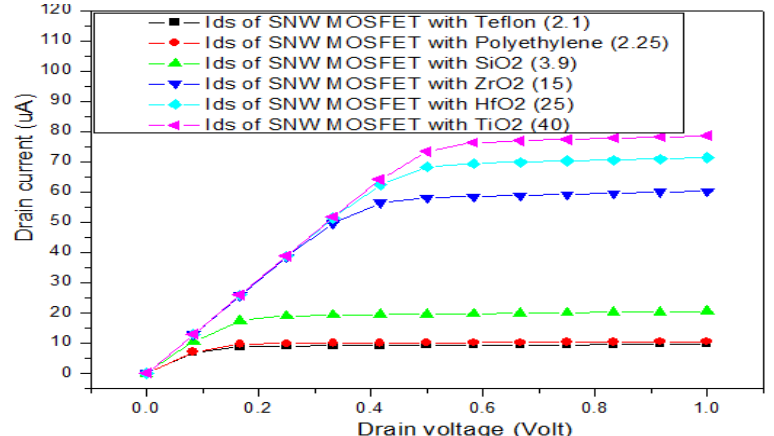

Fig.5. (b) Variation in Dielectric material

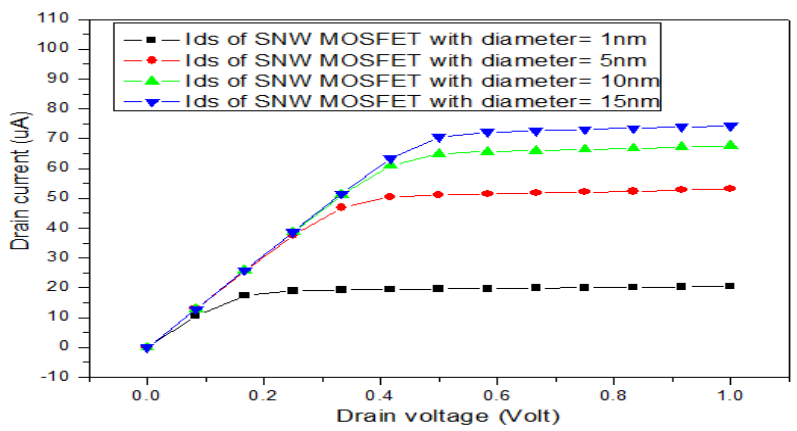

Fig.5. (d) Variation in SNW diameter 


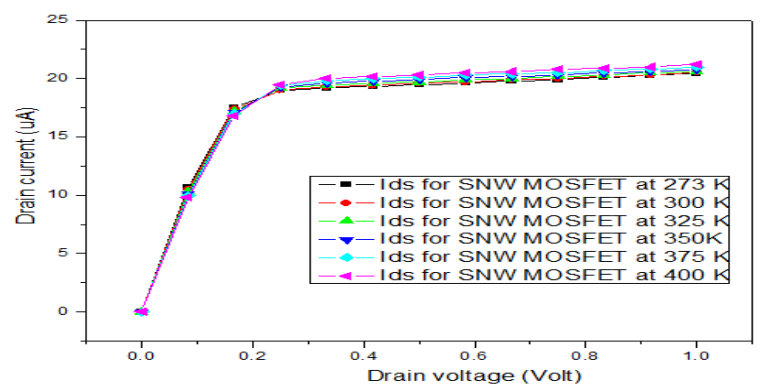

Fig.5. (e) Variation in temperature

The simulation results show that the drain current increases with increase in GCP, high-k dielectric materials, with decrease in dielectric thickness, with increase in SNW diameter and there is no appreciable variation for change in temperature.

\subsection{Drain Induced Barrier lowering}

Simulations were carried out to obtain Drain Induced Barrier lowering in a silicon nano wire MOSFET with gate voltage as $0-1 \mathrm{~V}$, drain voltage as $0-1 \mathrm{~V}$ and variations in input parameters as follows

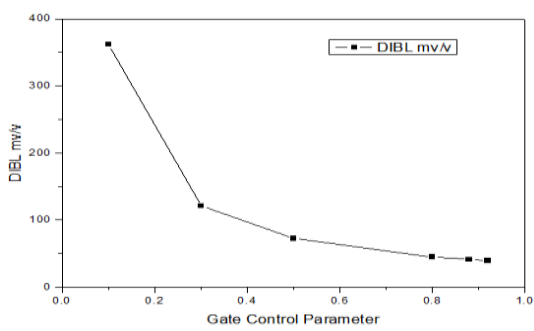

Fig.6. (a) Variation in GCP thickness

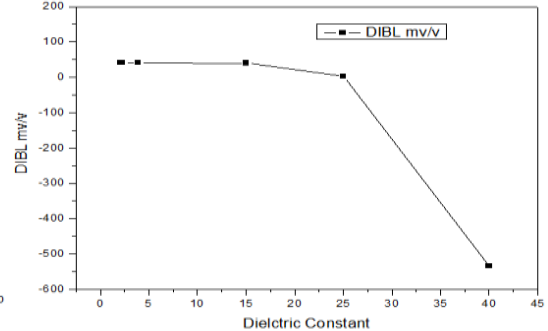

Fig.6. (b) Variation in Dielectric material

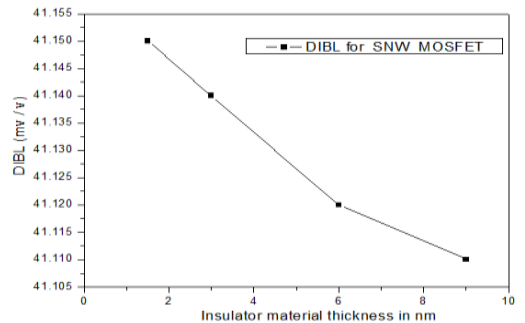

Fig.6. (c) Variation in Dielectric

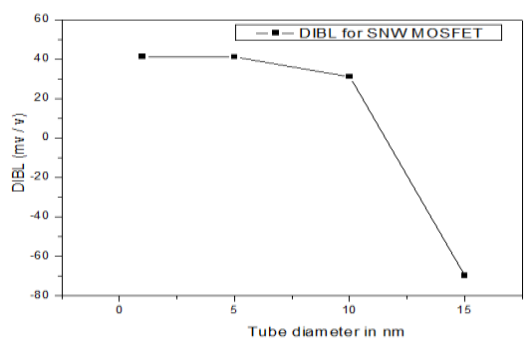

Fig.6. (d) Variation in SNW diameter

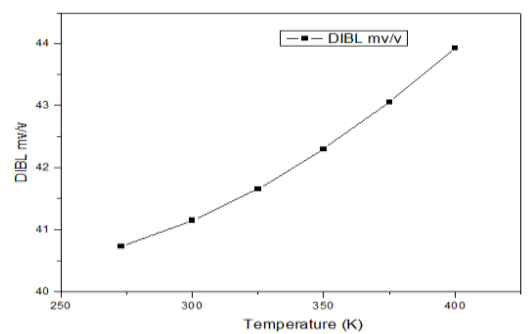

Fig.6. (e) Variation in temperature

The simulation results shows that the DIBL decreases with increase in GCP, high-k dielectric materials, increase in dielectric thickness, increase in SNW diameter and it increases when increase in temperature.

\subsection{Threshold swing}

Simulations were carried out to obtain Threshold swing in a silicon nano wire MOSFET with gate voltage as $0-1 \mathrm{~V}$ and drain voltage as $0-1 \mathrm{~V}$ and variations in input parameters as follows

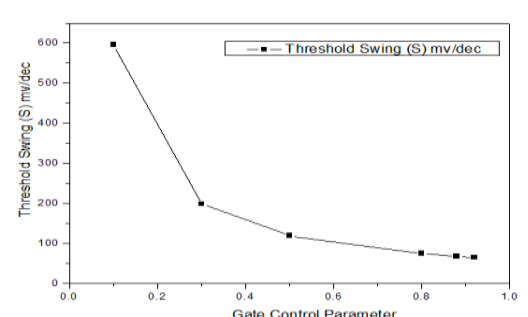

Fig.7. (a) Variation in GCP thickness

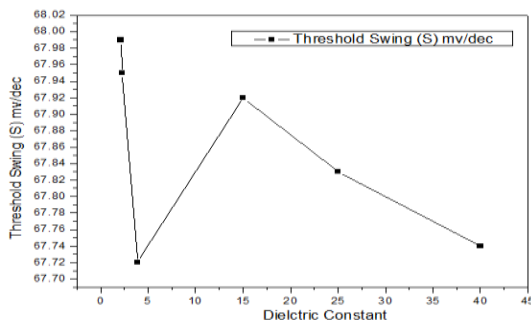

Fig.7. (b) Variation in Dielectric material

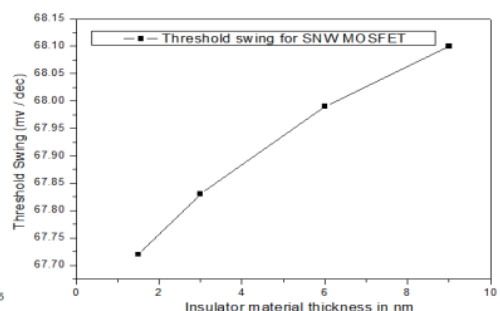

Fig.7. (c) Variation in Dielectric 


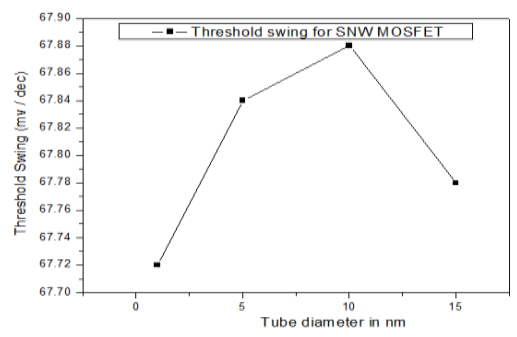

Fig.7. (d) Variation in SNW diameter

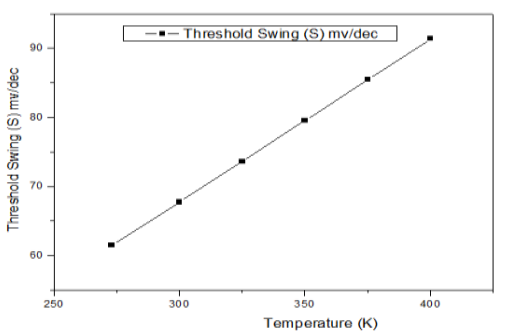

Fig.7. (e) Variation in temperature

The simulation results show that threshold swing decreases with increase in GCP and high-k dielectric materials. It increase for increase in dielectric thickness, increase in diameter (upto 10nm), with increase in temperature.

\subsection{Carrier injection velocity}

Simulations were carried out to obtain carrier injection velocity in a silicon nano wire MOSFET with gate voltage as $0-1 \mathrm{~V}$ and drain voltage as $0-1 \mathrm{~V}$ and variations in input parameters

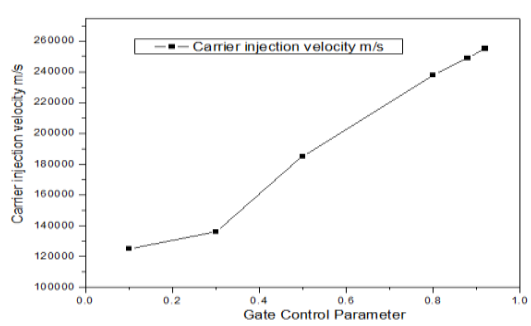

Fig.8. (a) Variation in GCP thickness

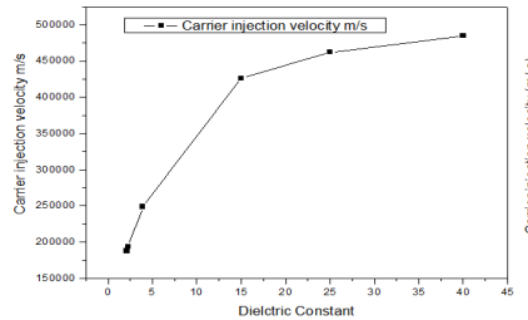

Fig.8. (b) Variation in Dielectric material

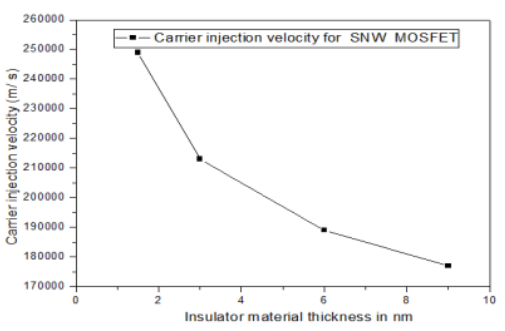

Fig.8. (c) Variation in Dielectric

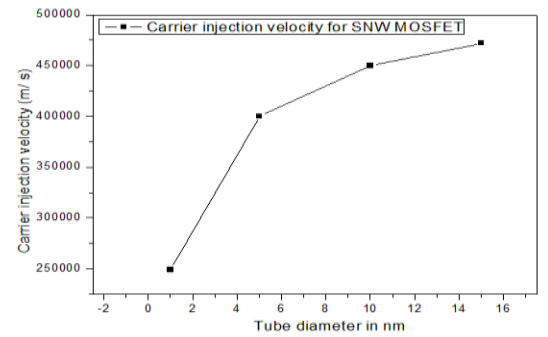

Fig.8. (d) Variation in SNW diameter

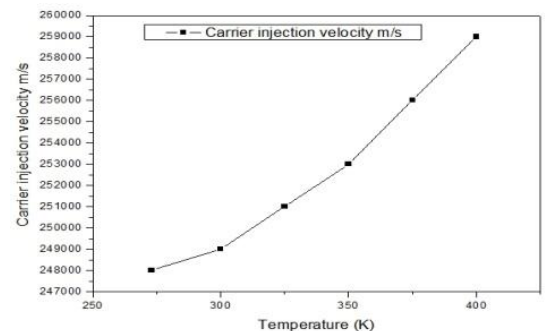

Fig.8. (e) Variation in temperature

The simulation results show that carrier injection velocity increases with increase in GCP, high-k dielectric materials, increase in SNW diameter, increase in temperature and decreases with increase in dielectric thickness.

\subsection{On current}

Simulations were carried out to obtain on current in a silicon nano wire MOSFET with gate voltage as $0-1 \mathrm{~V}$ and drain voltage as $0-1 \mathrm{~V}$ and variations in input parameters

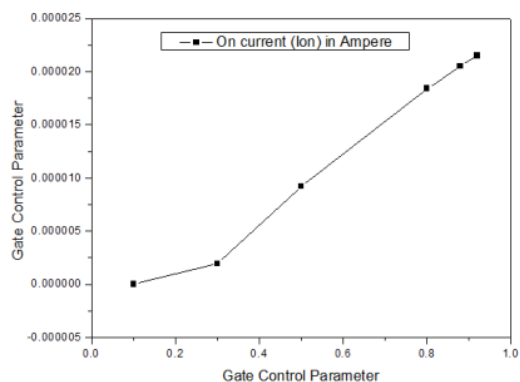

Fig.9. (a) Variation in GCP thickness

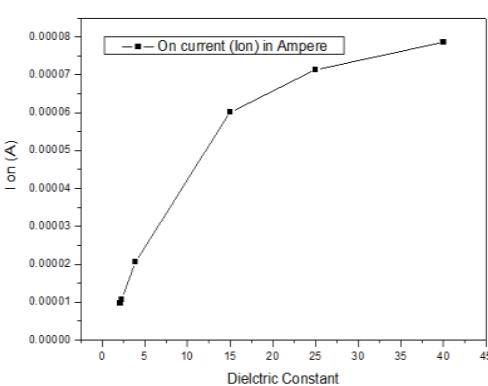

Fig.9. (b) Variation in Dielectric material

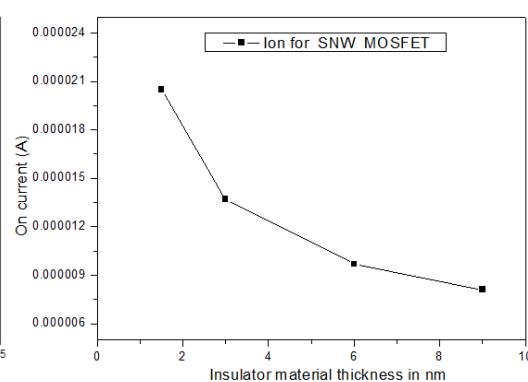

Fig.9. (c) Variation in Dielectric 


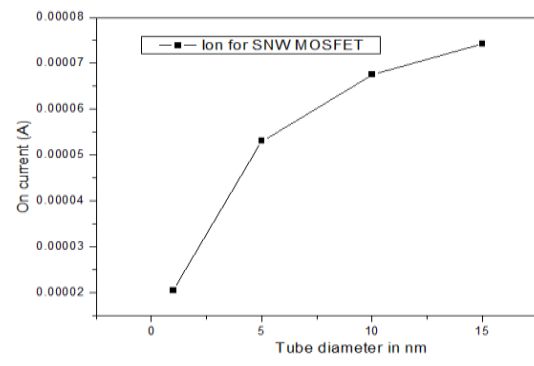

Fig.9. (d) Variation in SNW diameter

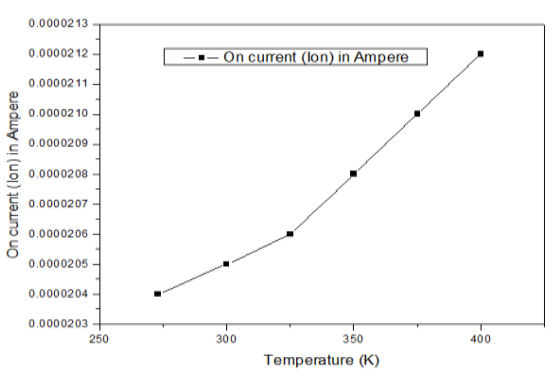

Fig.9. (e) Variation in temperature

The simulation results show that On current $\left(\mathrm{I}_{\mathrm{on}}\right)$ increases with increase in GCP, high-k dielectric materials, with increase in SNW diameter, with increase in temperature and decreases with increase in dielectric thickness.

\subsection{Off current}

Simulations were carried out to obtain off current in a silicon nano wire MOSFET with gate voltage as $0-1 \mathrm{~V}$ and drain voltage as $0-1 \mathrm{~V}$ and variations in input parameters
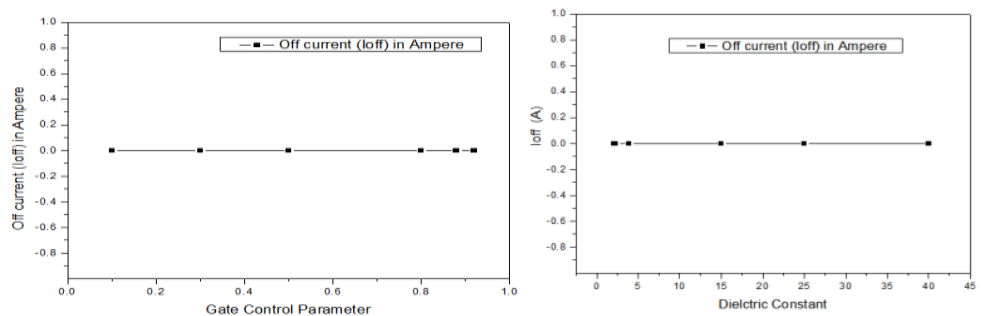

Fig.10. (b) Variation in Dielectric material

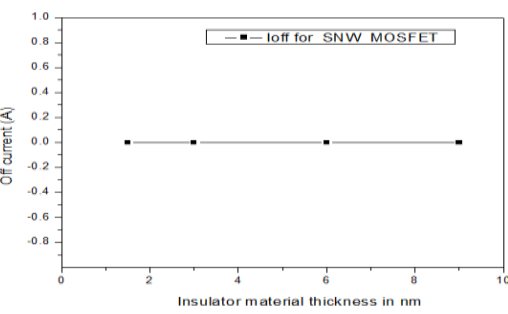

Fig.10. (c) Variation in Dielectric thickness

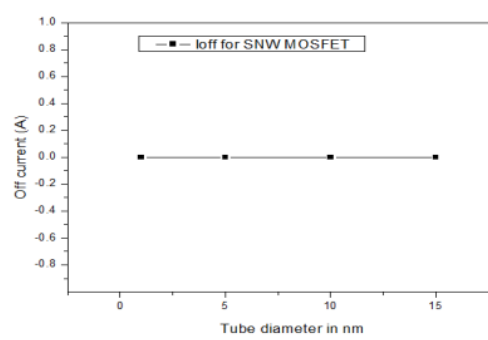

Fig.10. (d) Variation in SNW diameter

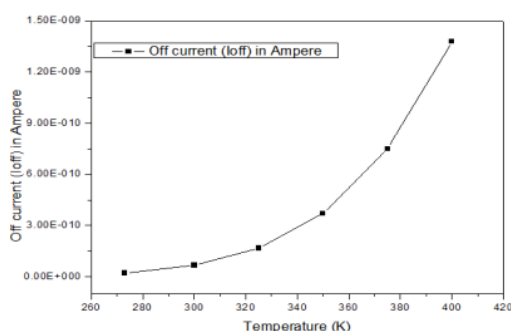

Fig.10. (e) Variation in temperature

The simulation results show that off current $\left(\mathrm{I}_{\text {off }}\right)$ increases with increase in temperature and no variation for change in GCP, dielectrics, dielectric thickness and SNW diameter.

\subsection{Output conductance}

Simulations were carried out to obtain output conductance in a silicon nano wire MOSFET with gate voltage as $0-1 \mathrm{~V}$ and drain voltage as $0-1 \mathrm{~V}$ and variations in input parameters

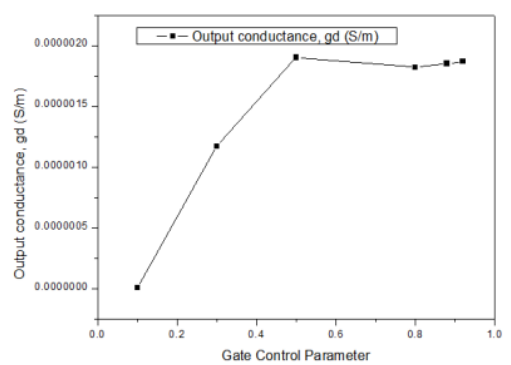

Fig.11. (a) Variation in GCP thickness

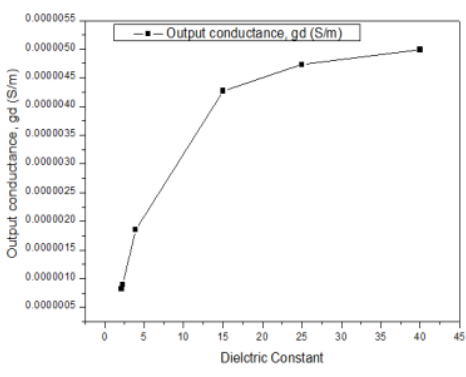

Fig.11. (b) Variation in Dielectric material

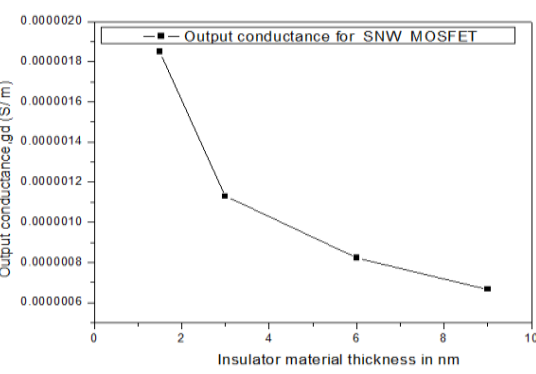

Fig.11. (c) Variation in Dielectric 


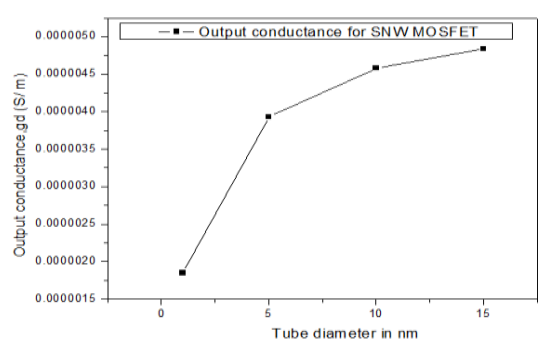

Fig.11. (d) Variation in SNW diameter

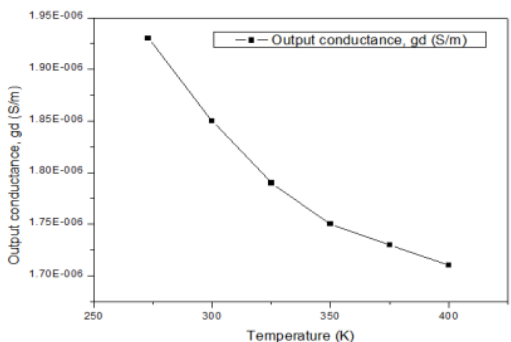

Fig.11. (e) Variation in temperature

The simulation results show that output conductance increases with increase in GCP, high-k dielectric materials, with increase in SNW diameter and decreases with increase in dielectric thickness, with increase in temperature.

\subsection{Transconductance}

Simulations were carried out to obtain transconductance in a silicon nano wire MOSFET with gate voltage as $0-1 \mathrm{~V}$ and drain voltage as $0-1 \mathrm{~V}$ and variations in input parameters

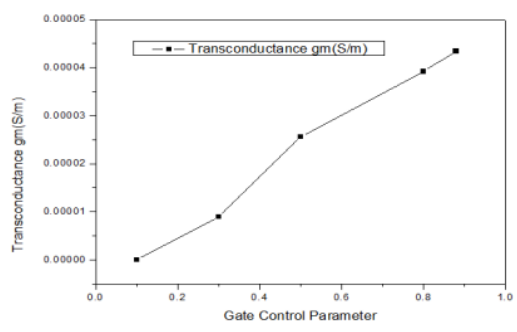

Fig.12. (a) Variation in GCP thickness

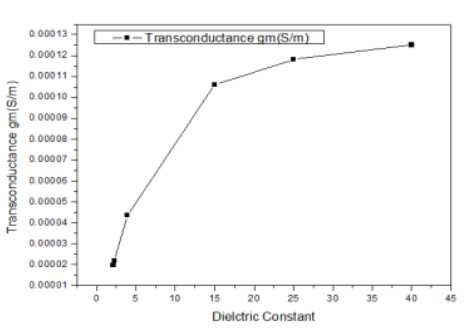

Fig.12. (b) Variation in Dielectric material

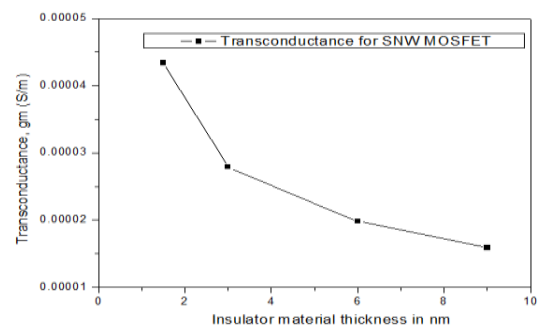

Fig.12. (c) Variation in Dielectric

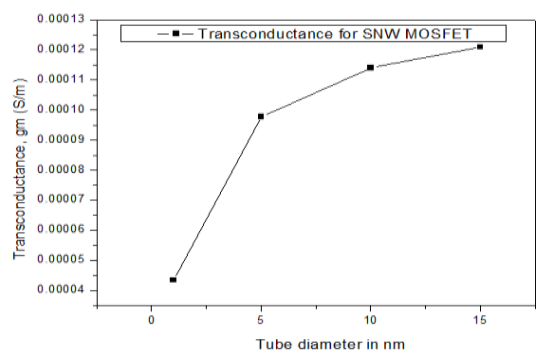

Fig.12. (d) Variation in SNW diameter

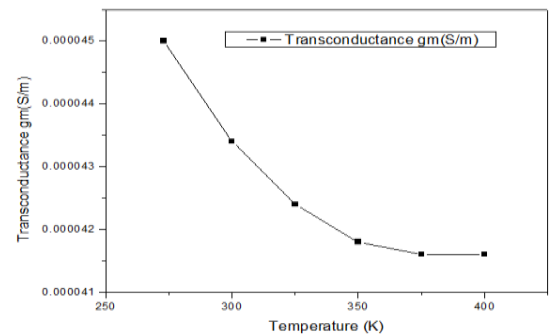

Fig.12. (e) Variation in temperature

The simulation results show that transconductance increases with increase in GCP, high-k dielectric materials, with increase in SNW diameter and decreases with increase in dielectric thickness, with increase in temperature.

\subsection{Voltage gain}

Simulations were carried out to obtain voltage gain in a silicon nano wire MOSFET with gate voltage as $0-1 \mathrm{~V}$ and drain voltage as $0-1 \mathrm{~V}$ and variations in input parameters

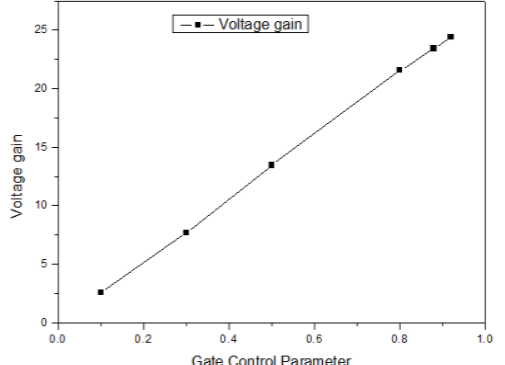

Fig.13. (a) Variation in GCP thickness

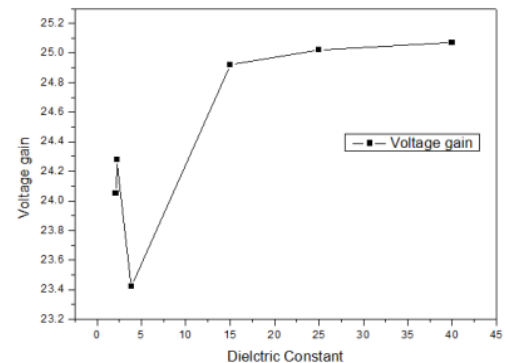

Fig.13. (b) Variation in Dielectric material

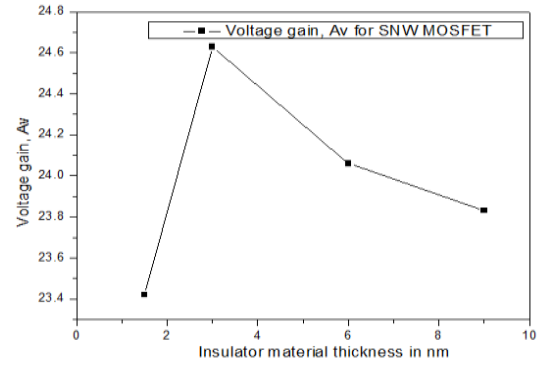

Fig.13. (c) Variation in Dielectric 


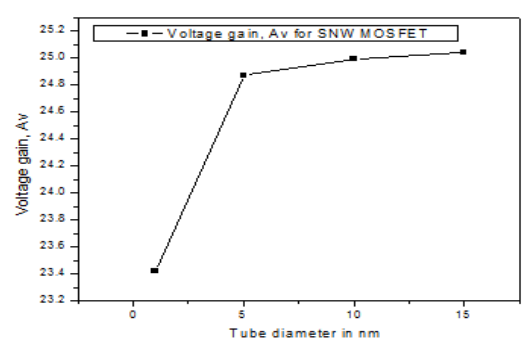

Fig.13. (d) Variation in SNW diameter

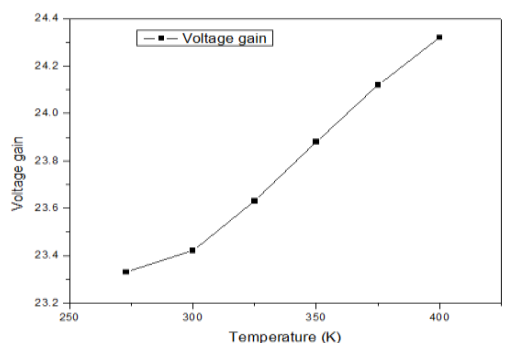

Fig.13. (e) Variation in temperature

The simulation results show that voltage gain increases with increase in GCP, high-k dielectric materials, with increase in SNW diameter, with increase in temperature and decreases with increase in dielectric thickness.

\section{Conclusions}

The various output parameters of silicon nanowire MOSFET were studied. From the discussions of the simulation results, we can conclude that in order to have better performance, the various input parameters should be high GCP, High-k dielectric materials, Small dielectric thickness, Large silicon nanowire diameter and low temperature is advisable.

\section{References}

[1] Dr.E.N.Ganesh, Kaushik Ragavan, Krishna Kumar "Study and simulation of silicon nanowire field effect transistor at subthreshold conditions using high k dielectric layer at room temperature" GESJ: Physics 2010

[2] Nagsen Meshram "Synthesis and characterization of silicon nanowire using Hot Wire Chemical Vapour deposition"

[3] Yi Cui, Zhaohui Zhong, Deli Wang, Wayne U. Wang, and Charles M. Lieber "High Performance Silicon Nanowire Field Effect Transistors" nano letters 2003

[4] www.nanohub.org 